\title{
Prognostic factors in pulmonary arterial hypertension: Literature review
}

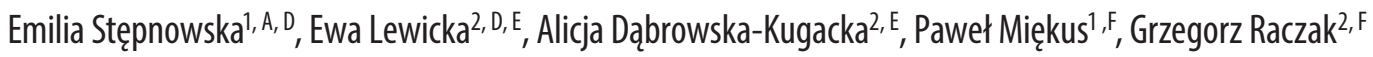 \\ ${ }^{1}$ Department of Cardiology, Saint Vincent de Paul Hospital, Gdynia, Poland \\ ${ }^{2}$ Department of Cardiology and Cardiac Electrotherapy, Medical University of Gdańsk, Poland \\ A - research concept and design; $B$ - collection and/or assembly of data; $C$ - data analysis and interpretation; \\ $D$ - writing the article; $E$ - critical revision of the article; $F$ - final approval of article
}

Address for correspondence

Emilia Stępnowska

E-mail: stepnowska.emilia@gmail.com

Funding sources

None declared

Conflict of interest

None declared

Received on 0ctober 25, 2015

Revised on December 09,2015

Accepted on February 19, 2016

\begin{abstract}
Pulmonary arterial hypertension is a disease that has a bad influence on the patient's prognosis. Recently, the possibility of therapy has dramatically changed. Nowadays, the treatment of this disease is concerned mainly with the pathophysiological target. In clinical practice, it is important to start therapy at the appropriate time, when the patient is qualified because of an unsatisfactory examination result or improve therapy when the patient is getting worse. The understanding of prognosis factors in pulmonary arterial hypertension is necessary, because it is used to determine the length of patients' life expectancy. In September of 2015, new guidelines of ESC concerning the diagnosis and treatment of pulmonary hypertension have been presented. In our article we centered on the prognosis factor in pulmonary arterial hypertension. This document is a continuation of ESC guidelines. Many of the most contemporary articles are here summarized.
\end{abstract}

Key words: pulmonary arterial hypertension, prognosis, risk factor, mortality

DOI

10.17219/acem/61855

\section{Copyright}

Copyright by Author(s)

This is an article distributed under the terms of the

Creative Commons Attribution Non-Commercial License

(http://creativecommons.org/licenses/by-nc-nd/4.0/) 
Pulmonary hypertension means an abnormal increase in pulmonary arterial pressure, which may occur in various heart, lung, and pulmonary vascular diseases. Pulmonary hypertension is defined as the mean pulmonary arterial pressure $\geq 25 \mathrm{~mm} \mathrm{Hg}$ at rest as assessed by right heart catheterization (RHC). ${ }^{1}$ Values obtained during exercise are of no diagnostic significance.

The current classification includes 5 etiologic categories of pulmonary hypertension, and in the present review we focused on the first group, i.e. pulmonary arterial hypertension (PAH). ${ }^{1}$ This group includes patients with idiopathic PAH, hereditary forms of PAH (e.g. due to BMPR2 gene mutation), pulmonary hypertension due to connective tissue disease, HIV infection, or portal hypertension, and patients with uncorrected congenital heart disease (most commonly Eisenmenger syndrome).

$\mathrm{RHC}$ is required to confirm the diagnosis of pulmonary hypertension, and pressure measurements include the assessment of pulmonary arterial wedge pressure (PAWP) to differentiate between precapillary and postcapillary pulmonary hypertension. PAWP values below $15 \mathrm{~mm} \mathrm{Hg}$ indicate that a left heart disease is an unlikely cause of pulmonary hypertension. It should be noted that the lack of standardization of PCWP measurements has contributed to differences between various centers when measuring this parameter. ${ }^{2}$ The most recent guidelines recommend calculating the diastolic pressure gradient (DPG) as a difference between pulmonary artery diastolic pressure and PAWP in order to differentiate between isolated postcapillary pulmonary hypertension and combined pre- and postcapillary pulmonary hypertension. ${ }^{1}$ DPG values below $7 \mathrm{~mm} \mathrm{Hg}$ (with PAWP > $15 \mathrm{~mm} \mathrm{Hg}$ ) indicate the presence of isolated postcapillary pulmonary hypertension, most commonly due to a left heart disease.

\section{Prognosis}

Prognosis in PAH is severe and when untreated it is a potentially fatal disease that rapidly leads to disability and premature mortality. Evaluation of disease severity, including clinical assessment, imaging and haemodynamics, during follow-up visits is essential when selecting patients for specific drug therapy or its intensification. Knowledge of prognostic factors associated with poor outcomes is helpful in deciding on the correct treatment plan.

The type of disease underlying PAH has an important role when determining prognosis in these patients. In patients with congenital heart disease, prognosis is better compared to those with idiopathic PAH. ${ }^{3}$ In patients with congenital cardiac shunt disease who have not been treated surgically, a right-to-left shunt reduces right ventricular pressure, which has a protective effect on the right ventricular function. Recent studies indicate a very poor prognosis in patients with PAH that developed fol- lowing surgical correction of a congenital heart disease compared to patients with Eisenmenger syndrome. ${ }^{4}$

Prognosis is also very poor in patients with systemic sclerosis in whom pulmonary vascular smooth muscle proliferation and constriction leads to PAH. ${ }^{5}$ Pulmonary hypertension is a major cause of death in this group in addition to pulmonary fibrosis. Before the era of vasodilating drug therapy, the median survival of patients with systemic sclerosis and PAH was $1-3$ years. ${ }^{5}$ Concomitant interstitial lung disease adds to this poor prognosis, resulting in a 5-fold increase in mortality. One-, 2-, and 3 -year survival in patients with $\mathrm{PAH}$ and interstitial lung disease was 82,46 , and $39 \%$, respectively, compared to 87 , 79 , and $64 \%$ in patients without interstitial lung disease. ${ }^{6}$

\section{Prognostic factors}

To analyze the effect of age and gender on prognosis in patients with $\mathrm{PAH}$, Corciova et al. performed a prospective study that included 553 patients diagnosed with pulmonary hypertension, including idiopathic PAH. ${ }^{3}$ There were no differences in mortality in relation to gender in this group. Also patient age, which was on average 64.7 years at the time of death, was not found to be a risk factor for mortality. In contrast, another study showed that male gender and older age had a significant influence on survival. ${ }^{7}$ Patients with idiopathic PAH below 14 years of age and above 65 years of age have worse prognosis compared to those aged 14-65 years. However, children seem to show increased vasodilatation in response to a rise in pulmonary arterial pressure and thus are characterized by a better prognosis. ${ }^{8}$

Mortality risk is closely related to the disease severity as categorized using the World Health Organization (WHO) functional classification and is highest in the WHO class IV. ${ }^{9}$ Before the era of specialist treatment, the mean survival was about 6 months in the WHO class IV, 2.5 years in the WHO class III, and 6 years in the WHO class I. The difference in survival between WHO class III and IV patients was significant ( $\mathrm{p}<0.001)$. Progression to a higher WHO class is one of the strongest adverse prognostic factors and should prompt a search for a reason of such worsening. ${ }^{10}$

In addition to the WHO class, exercise tolerance in patients with PAH is assessed on the basis of the 6-min walking distance (6MWD). During this test, aside from the walking distance, dyspnea severity by the Borg scale, heart rate, systemic blood pressure, and oxygen saturation are also evaluated. Savarese et al. performed a metaanalysis of 22 randomized clinical trials to determine the relationship between 6MWD and the risk of a combined clinical endpoint that included death, hospitalization due to $\mathrm{PAH}$, and the need for lung or lung and heart transplantation. ${ }^{11}$ This analysis included studies with documented 6MWD at baseline and at follow-up or at 
the time of clinical endpoint occurrence. No association was found between the change in 6MWD at follow-up compared to baseline and the risk of clinical endpoint occurrence.

Performing a 6-min walking test is a useful way to evaluate treatment effectiveness. The prognostic value of this parameter lies not in the change of 6MWD in response to treatment, but most of all in its absolute value, particularly if it is lower than $250 \mathrm{~m} .^{7}$ There was significantly lower mortality in patients with 6MWD higher than $440 \mathrm{~m} .^{12}$ A number of studies showed that an improvement in the 6-minute walking test was parallel to the improvement of hemodynamic parameters as assessed during cardiac catheterization. ${ }^{11}$ A significant association between the change in 6MWD and the change in pulmonary vascular resistance measured during $\mathrm{RHC}$ was found. ${ }^{11}$ However, monitoring PAH on the basis of 6MWD is significantly limited by lack of standardization, suboptimal measurement reproducibility, and the effect of gender, height, age, and patient motivation on 6MWD values. Despite this, evaluation of 6MWD is the only exercise test to assess the effectiveness of PAH treatment that was approved by the Food and Drug Administration (FDA) and the European Medicines Agency (EMA).

Echocardiography is commonly used for diagnostic and treatment monitoring purposes in patients with $\mathrm{PAH}$ due to its wide availability, non-invasive nature, and reproducibility. An adverse prognostic sign is the presence of pericardial effusion, which accumulates due to impaired venous and lymphatic drainage caused by right ventricular systolic dysfunction and a subsequent increase in right atrial pressure. ${ }^{21}$ However, the threshold amount of pericardial fluid, which is associated with significantly worsened prognosis in patients with $\mathrm{PAH}$, has not been determined yet.

A parameter that correlates with right ventricular ejection fraction is tricuspid annular plane systolic excursion (TAPSE). ${ }^{22}$ TAPSE values below $18 \mathrm{~mm}$ indicate right ventricular systolic dysfunction and impaired remodeling, leading to worse outcomes. ${ }^{22}$ Of note, the evaluation of TAPSE is not useful when a significant tricuspid regurgitation is present. In the study by Ghio et al. performed in a group of patients with PAH, TAPSE below $15 \mathrm{~mm}$ was associated with higher mortality compared to patients with TAPSE above $15 \mathrm{~mm} .^{23}$

An increase in the Tei index of myocardial performance, calculated as the sum of isovolumic contraction and relaxation times divided by the right ventricular ejection time, is observed alongside the decline in the right ventricular function. The Tei index is significantly increased in patients with $\mathrm{PAH}$, and its values of 0.83 or higher indicate poor prognosis. ${ }^{24}$ However, an evaluation of the Tei index for the right ventricle has some limitations such as high dependence on the volume status, poor measurement reliability during tachycardia, and pseudonormalization in very advanced right ventricular failure, which limits the ability to use this parameter in all patients.

Another study that included 72 patients with idiopathic PAH, Ghio et al. showed that mortality was higher in patients with the right ventricular dimension $>36.5 \mathrm{~mm}$ by echocardiography compared to those with lower values ( $\mathrm{p}=0.044) .{ }^{25}$ Right ventricular free wall thickness was not found to have an influence on survival. However, when both these parameters are taken into account, the right ventricular dimension $>36.5 \mathrm{~mm}$ combined with the right ventricular free wall thickness $>6.6 \mathrm{~mm}$ was not associated with a worse prognosis. This may be explained by the fact that the risk is reduced if right ventricular dilatation, indicating poor prognosis, is accompanied by an increase in wall thickness. In other words, an increase in the right ventricular wall thickness is protective when the right ventricle is dilated, as myocardial wall tension is then reduced. Better right ventricular function, and thus better prognosis, is also indicated by an increase in the right ventricular systolic pressure by $30 \mathrm{~mm} \mathrm{Hg}$ during stress echocardiography, indicating preserved right ventricular contractility reserve. ${ }^{26}$

In turn, Bustamante-Labart et al. showed a relationship between the right atrial size and the risk of death or the need for heart or heart and lung transplantation in patients with idiopathic PAH. ${ }^{27}$ Death or transplantation occurred in $81 \%$ of patients with the right atrial area above $27 \mathrm{~cm}^{2}$, including all patients with the right atrial area higher than $34 \mathrm{~cm}^{2}$. Right atrial area $>26 \mathrm{~cm}^{2}$ was also indicated in the most recent guidelines as associated with worse prognosis. ${ }^{1}$

Biochemical markers are also used to evaluate prognosis in patients with PAH. Their advantages include easy repeatability and a non-invasive nature. Serum uric acid level was found to be of a significant prognostic importance in patients with heart failure, as it reflects the degree of aerobic metabolism impairment in ischemic tissues. Bendayan et al. showed a correlation between serum uric acid level and the severity of pulmonary hypertension in patients with $\mathrm{PAH} .{ }^{13}$ A positive association was found between serum uric acid concentration and the severity of heart failure by the WHO classification $(\mathrm{p}<0.01)$, and a negative association for the 6 -min walk test: the higher was serum uric acid level, the lower was 6MWD. Serum uric acid concentration was also found to be significantly higher in those patients who died during the follow-up compared to survivors. However, no association was found between serum uric acid level and patient age, nor the mean pulmonary arterial pressure, cardiac output, and mixed venous oxygen saturation assessed during RHC. Nagaya et al. showed a positive correlation between serum uric acid concentration and an increase in pulmonary vascular resistance. ${ }^{14}$ In addition, a trend for serum uric acid level lowering was seen in response to vasodilating drug therapy. In contrast, no association was found between arterial oxygen saturation and 
serum uric acid concentration, despite the fact that other studies indicated the activation of the purine degradation pathway in ischemic tissues, resulting in the overproduction of uric acid. ${ }^{15}$ Clearly, the effect of other factors on serum uric acid level should be taken into account when evaluating prognosis in patients with $\mathrm{PAH}$, such as concomitant diseases (chronic obstructive pulmonary disease and chronic kidney disease), diet, and medications (diuretics, allopurinol).

Brain natriuretic peptide (BNP) or inactive, more stable $\mathrm{N}$-terminal pro-B-type natriuretic peptide (NT-proBNP) are also parameters of prognostic importance in patients with heart failure. BNP and NT-proBNP are released by right and left ventricular cardiomyocytes in response to an increase in wall tension. In a group of patients with idiopathic PAH, Nagaya et al. showed that BNP concentration above $150 \mathrm{pg} / \mathrm{mL}$ was associated with adverse prognosis. ${ }^{16}$ For NT-proBNP level, the threshold value for adverse prognosis was $1400 \mathrm{pg} / \mathrm{mL} \cdot{ }^{17}$ In the study by Blyth et al., cardiac magnetic resonance imaging (MRI) was performed and NT-proBNP concentration was measured in a group of 25 patients with pulmonary hypertension (including 19 with PAH).$^{18}$ A negative relationship was found between NT-proBNP level and right ventricular ejection fraction. NT-proBNP level equal to or higher than $1685 \mathrm{pg} / \mathrm{mL}$ was nearly 100\% sensitive and specific for right ventricular systolic failure. Similarly, NT-proBNP concentration in the study by Fijałkowska et al. was found to correlate with hemodynamic and echocardiographic parameters of right ventricular systolic dysfunction. ${ }^{17}$ In early stage of the right ventricular failure, no signs may be identified during physical examination. Natriuretic peptide level measurements in patients with PAH may lead to earlier institution of specific drug therapy and thus prevent progression to severe disease. In clinical practice, NT-proBNP levels are measured more frequently than BNP levels, as NT-proBNP is more stable both in vivo and in vitro.

Torbicki et al. evaluated high-sensitivity troponin $\mathrm{T}$ (hs-TnT) concentration in 56 patients with pulmonary hypertension (including 51 with PAH). The mean hs-TnT level was $0.034 \mathrm{ng} / \mathrm{mL}$ (range $0.010-0.077 \mathrm{ng} / \mathrm{mL}$ ). ${ }^{19} \mathrm{He}$ modynamic parameters evaluated during cardiac catheterization did not differ between patients with elevated or normal hs-TnT levels, but patients with higher hs-TnT concentrations were characterized by a significantly higher heart rate, lower mixed venous oxygen saturation, higher NT-proBNP concentration, and lower exercise tolerance during the 6-min walking test. During 2-year follow-up, 63\% patients with elevated hs-TnT level died compared to $15 \%$ among those with normal hs-TnT concentration. These studies indicate the importance of hsTnT level measurements during follow-up visits in patients with PAH.

Takeda et al. evaluated the effect of hepatic dysfunction on prognosis in patients with $\mathrm{PAH} .{ }^{20}$ Hepatic dysfunction often accompanies right ventricular failure and results from passive hyperemia and reduced hepatic blood flow. In the cited study that included 62 WHO class II-IV patients with PAH (idiopathic or due to connective tissue disease), an elevated bilirubin level was found to be associated with higher disease severity. Patients with elevated total bilirubin level remained in a higher WHO class and had higher NT-proBNP levels and higher right atrial pressures as measured during cardiac catheterization. In addition, bilirubin was found to be a more sensitive marker of right ventricular failure compared to transaminase levels.

Cardiac MRI is increasingly commonly used in patients with PAH, particularly for monitoring the disease course. This modality allows highly precise three-dimensional evaluation of cardiac morphology and unlike echocardiography, it is not limited by suboptimal acoustic windows. Unfortunately, limitations of MRI include low availability and a relatively high cost. In the study by Bradlow et al. the right to left ventricular mass ratio as evaluated by MRI was associated with survival in patients with PAH. ${ }^{28}$ The ventricular mass index (VMI) or the end-diastolic ratio of right to left ventricular mass higher than 0.7 and an increased right ventricular mass were indicators of poor prognosis. In the study by van Wolferen et al., adverse prognostic MRI parameters included right ventricular dilatation, reduced right ventricular ejection fraction and ejection volume, and reduced left ventricular end-diastolic volume. ${ }^{29}$

\section{Summary}

Pulmonary arterial hypertension is a condition associated with particularly poor prognosis. Thus, identification of its prognostic factors is of major importance for therapeutic decision making, including treatment intensification.

Evaluation of prognosis in patients with PAH has been discussed in the most recent 2015 European Society of Cardiology/European Respiratory Society guidelines on the diagnosis and management of pulmonary hypertension. ${ }^{1}$ Depending on one-year mortality risk, patients with PAH were divided into 3 groups characterized by a high (> 10\%), intermediate $(5-10 \%)$, or low risk $(<5 \%)$. A number of factors including the clinical presentation of PAH, 6MWD, BNP/NT-proBNP levels, and echocardiographic, spiroergometric, and RHC parameters are taken into account when evaluating prognosis.

As indicated by the authors, rapid disease progression, disease severity (WHO class IV), clinically overt right ventricular failure, 6MWD $<165 \mathrm{~m}$, and syncope are all associated with an increased mortality risk $(>10 \%$ at one-year follow-up). Adverse prognostic factors also include BNP level > $300 \mathrm{ng} / \mathrm{L}$ or NT-proBNP level $>1400$ $\mathrm{ng} / \mathrm{L}$, pericardial effusion, right atrial size $>26 \mathrm{~cm}^{2}$ by 
echocardiography, peak oxygen consumption $\left(\mathrm{VO}_{2} \max \right)$ $<11 \mathrm{~mL} / \mathrm{min} / \mathrm{kg}$ (<35\% of the predicted value) by the spiroergometric testing, and right atrial pressure $>14$ $\mathrm{mmHg}$, cardiac output index $<2 \mathrm{~L} / \mathrm{min} / \mathrm{m}^{2}$ and mixed venous oxygen saturation $<60 \%$ by RHC.

In contrast, better prognosis (one-year mortality risk $<5 \%$ ) is indicated by a lower disease severity (WHO class I-II) and stable clinical course, no signs of right ventricular failure in the clinical examination, 6MWD $>440 \mathrm{~m}$, BNP level $<50 \mathrm{ng} / \mathrm{L}$ or NT-proBNP level $<300 \mathrm{ng} / \mathrm{L}$, no pericardial effusion, normal right atrial size $\left(<18 \mathrm{~cm}^{2}\right)$ by echocardiography, $\mathrm{VO}_{2} \max >15 \mathrm{~mL} / \mathrm{min} / \mathrm{kg}$ ( $>65 \%$ of the predicted value) by the spiroergometric testing, and normal right atrial pressure $(<8 \mathrm{~mm} \mathrm{Hg})$, cardiac output index $>2.5 \mathrm{~L} / \mathrm{min} / \mathrm{m}^{2}$ and mixed venous oxygen saturation $>65 \%$ by $\mathrm{RHC}$.

Knowledge of prognostic factors in PAH allows for the identification of therapeutic targets in this patient group. Treatment efforts in a patient with PAH should aim at obtaining the above defined low risk clinical profile, which indicates better outcomes and thus better effectiveness of the therapy that has been instituted.

\section{References}

1. Galie N, Humbert M, Vachiery JL, et al. 2015 ESC/ERS Guidelines for the diagnosis and treatment of pulmonary hypertension. Eur Heart J. 2015. pii: ehv317.

2. Hoeper MM, Bogaard HJ, Condliffe R, et al. Definitions and diagnosis of pulmonary hypertension. Turk Kardiyol Dern Ars. 2014;42 (Suppl 1):55-66.

3. Corciova FC, Arsenescu-Georgescu C. Prognostic factors in pulmonary hypertension. Maedica (Buchar). 2012;7(1):30-37.

4. Manes $A$, Palazzini $M$, Leci $E$, et al. Current era survival of patient with pulmonary arterial hypertension associated with congenital heart disease: A comparison between clinical subgroups. Eur Heart J. 2014;35(11):716-724.

5. York M, Faber HW. Pulmonary hypertension: Screening and evaluation in scleroderma. Curr Opin Rheumatol. 2011;23(6):536-544.

6. Math SC, Hummers LK, Champion HC, Wigley FM, Zaiman A. Survival in pulmonary hypertension associated with the scleroderma spectrum of diseases: Impact of interstitial lung disease. Arthritis Rheum. 2009;60(2):569-577.

7. Benza RL, Miller DP, Gomberg-Maitland M, et al. Predicting survival in pulmonary arterial hypertension: Insights from the registry to evaluate early and long-term pulmonary arterial hypertension disease management (REVEAL). Circulation. 2010;122(2):164-172.

8. Haworth SG, Hislop AA. Treatment and survival in children with pulmonary arterial hypertension: The UK Pulmonary Hypertension Service for Children 2001-2006. Heart. 2009;95(4):312-317.

9. D'Alonzo GE, Barst RJ, Ayres SM, et al. Survival in patient with primary pulmonary hypertension. Results from a national prospective registry. Ann Intern Med. 1991;115(5):343-349.

10. Nickel N, Golpon H, Greer M, et al. The prognostic impact of follow up assessments in patients with idiopathic pulmonary arterial hypertension. Eur Respir J. 2012;39(3):589-596.
11. Savarese G, Paolillo S, Costanzo P, et al. Do changes of 6-minute walk distance predict clinical events in patients with pulmonary arterial hypertension? A meta-analysis of randomized trials. J Am Coll Cardiol. 2012;60(13):1192-1201.

12. Farber HW, Miller DP, McGoon MD, Frost AE, Benton WW. Predicting outcomes in pulmonary arterial hypertension based on the 6-minute walk distance. J Heart Lung Transplant. 2015;34(3):362-368.

13. Bendayan $D$, Shitrit $D$, Ygla $M$, et al. Hyperuricemia as a prognostic factor in pulmonary arterial hypertension. Respir Med. 2003;97(2):130-133.

14. Nagaya $N$, Uematsu $M$, Satoh $T$, et al. Serum uric acid levels correlate with the severity and the mortality of primary pulmonary hypertension. Am J Respir Crit Care Med. 1999;160(2):487-492.

15. Hasday JD, Grum CM. Nocturnal increase of urinary uric acid:creatinine ratio: A biochemical correlate of sleep-associated hypoxemia. Am Rev Respir Dis. 1987;135(3):534-538.

16. Nagaya N, Nishikimi T, Uematsu M, et al. Plasma brain natriuretic peptide as a prognostic indicator in patients with primary pulmonary hypertension. Circulation. 2000;102(8):865-870.

17. Fijalkowska A, Kurzyna M, Torbicki A, Szewczyk G, Florczyk M. Serum $\mathrm{N}$-terminal brain natriuretic peptide as a prognostic parameter in patients with pulmonary hypertension. Chest. 2006;129(5):1313$-1321$.

18. Blyth KG, Groenning BA, Mark PB, et al. NT-proBNP can be used to detect right ventricular systolic dysfunction in pulmonary hypertension. Eur Respir J. 2007;29(4):737-744.

19. Torbicki A, Kurzyna M, Kuca P, et al. Detectable serum cardiac tropo$\operatorname{nin} T$ as a marker of poor prognosis among patients with chronic pre capillary pulmonary hypertension. Circulation. 2003;108(7):844-848.

20. Takeda $Y$, Takeda $Y$, Tomimoto $S$, et al. Bilirubin as a prognostic marker in patients with pulmonary arterial hypertension. $B M C$ Pulm Med. 2010;10:22.

21. Eysmann SB, Palevsky HI, Reichek N, Hackney K, Douglas PS. Twodimensional and doppler echocardiographic and cardiac catheterization correlates of survival in primary pulmonary hypertension. Circulation. 1989;80(2):353-360.

22. Howard LS. Prognostic Factors in pulmonary arterial hypertension: Assessing the course of the disease. Eur Respir Rev. 2011;20(122):236-242.

23. Ghio S, Klersy C, Margin G, et al. Prognostic relevance of the echocardiographic assessment of right ventricular function in patients with idiopathic pulmonary arterial hypertension. Int J Cardiol. 2010;140(3):272-278.

24. Stone $A C$, Klinger JR. The right ventricle in pulmonary hypertension. In: Contemporary cardiology: Pulmonary hypertension. Red.: NS Hill I HW Farber. Totowa, NJ: Humana Press. 2008:93-125.

25. Ghio S, Pazzano AS, Klersy C, et al. Clinical and prognostic relevance of echocardiographic evaluation of right ventricular geometry in patients with idiopathic pulmonary arterial hypertension. Am J Cardiol. 2011;107(4):628-632.

26. Grunig E, Tiede H, Enyimayew EO, et al. Assessment and prognostic relevance of right ventricular contractile reserve in patients with severe pulmonary hypertension. Circulation. 2013;128(18):2005-2015.

27. Bustamante-Labarta M, Perrone S, de la Fuente RL, et al. Right atrial size and tricuspid regurgitation severity predict mortality or transplantation in primary pulmonary hypertension. J Am Soc Echocardiogr. 2002;15(10 Pt 2):1160-1164.

28. Bradlow WM, Assomull RG, Williams F, et al. Cardiovascular magnetic resonance measured right ventricular mass influences prognosis in pulmonary arterial hypertension. Eur Heart J. 2010;31:8.

29. Van Wolferen SA, Marcus JT, Boonstra A, et al. Prognostic value of right ventricular mass, volume and function in idiopathic pulmonary arterial hypertension. Eur Heart J. 2007;28(10):1250-1257. 